\title{
Distributed Carrier Aggregation in Small Cell Networks: A Game-theoretic Approach
}

\author{
Yuanhui Zhang, ${ }^{1,2}$, Chunrong $\mathrm{Kan}^{1}$, Kun $\mathrm{Xu}^{3}$ and Yuhua $\mathrm{Xu}^{1}$ \\ ${ }^{1}$ PLA University of Science and Technology, China \\ ${ }^{2}$ Zhenjiang Watercraft College, China \\ ${ }^{3}$ PLA Academy of National Defense Information, Wuhan, China \\ [e-mail: zyh0126@foxmail.com, kcr72@263.net, xukunown@163.com, yuhuaenator@gmail.com] \\ *Corresponding author: Yuhua Xu
}

Received May 1, 2015; revised September 15, 2015; accepted September 30, 2015;

published December 31, 2015

\begin{abstract}
In this paper, we investigate the problem of achieving global optimization for distributed carrier aggregation (CA) in small cell networks, using a game theoretic solution. To cope with the local interference and the distinct cost of intra-band and inter-band CA, we propose a non-cooperation game which is proved as an exact potential game. Furthermore, we propose a spatial adaptive play learning algorithm with heterogeneous learning parameters to converge towards NE of the game. In this algorithm, heterogeneous learning parameters are introduced to accelerate the convergence speed. It is shown that with the proposed game-theoretic approach, global optimization is achieved with local information exchange. Simulation results validate the effectivity of the proposed game-theoretic CA approach.
\end{abstract}

Keywords: Carrier aggregation, small cell network, game theory, inter-band cost

This research was supported by the National Science Foundation of China under Grant No.61401508. 


\section{Introduction}

$\mathbf{S}_{\text {mall cell is the most promising approach for providing a thousand-fold mobile traffic over }}$ the next decade [1]. Compared to the macrocell, small cell has lower power and smaller coverage areas. They can operate in licensed and unlicensed spectrum. Thus, more spectrum bands can be utilized by small cells, e.g., Super high frequency (SHF) and Extremely high frequency (EHF). However, as exponential growth of the number of devices and high data rate in future mobile communication systems [2]-[4], the demand of high bandwidth for high data rate is urgent. Carrier aggregation (CA) allows a cell to aggregate multiple channels for a wider bandwidth to transmit simultaneously. It is necessary to exploite CA to increase the data rate in small cell networks [5]-[6]. Thus, it is promising important to study the problem of resource allocation for distributed CA in dense small cell networks.

Actually, to be different from traditional cellular system, channel allocation for distributed CA in dense small cell networks faces a challenge that the mutual interference is more serious. Moreover, the impact caused by the distinct cost of intra-band and inter-band CA should be considered. The reason is that inter-band CA needs multiple FFT and multiple RF front-end in contrast that intra-band needs only one [7]. For the problem of distributed CA in small cell networks, the key task is to determine the aggregated channels among multiple small cells, which is a combinatorial majorization problem and hard to solve.

Generally, there are centralized gateways [8] in small cell networks that make centralized optimization possible. If we apply a centralized optimization to address this problem, e.g., [9]-[11], as the deployment of small cells become dense, this optimization problem is extremely complicated. For example, consider a network with 20 small cells and two band with two channels in each band. Consider a simple scenario in which each small cell aggregates at most two channels for CA, the corresponding number of all possible strategy selection profiles is $10^{20}$, which is hardly possible to achieve the optimal solutions. To solve this problem, we resort to game theory, which is a powerful optimization tool involving multiple interactive decision-makers [12]-[13]. In this paper we propose a game-theoretic solution for solving the problem of distributed CA in small cell networks. To the best of our knowledge, such an investigation has not been addressed effectively in existing researches.

To summarize, the main contributions of this paper are as follows:

1) We formulate the problem of distributed CA in small cell networks as a non-cooperative game. In particular, the utility function in the game takes into account local interference and the distinct cost of intra-band and inter-band CA. It is proved that the game is an exact potential game with the defined potential function serving as the potential function. It is shown that the strategy profile of achieving the optimal potential function can make the network throughput better.

2) We propose a spatial adaptive play algorithm with heterogeneous learning parameters (SAP-H) to converge towards Nash equilibrium (NE) of the game. In this algorithm, we introduce heterogeneous learning parameters to accelerate the convergence speed. Thus, compared with the conventional SAP algorithm, the proposed algorithm converges rapidly while keeping the network capacity. Moreover, the performance of the proposed SAP-H is close to the best Nash equilibria. 
3) We compare the average throughput for each small cell when varying the available spectrum resource and the degree of flexibility in performing CA respectively. We draw some meaningful conclusions.

The rest of the article is organized as follows. In Section 2, we present the related work. In Section 3, we give the system model and formulate the problem. In Section 4, we model the problem of CA as a non-cooperative game and investigate the properties of its NE. And we propose a spatial adaptive play algorithm with heterogeneous learning parameters. In Section 5 , simulation results are presented and conclusion is drawn in Section 6.

\section{Related Work}

The problem of CA was studied in some works. The authors studied the UE energy saving by applying carrier aggregation in [14]. In [15] a novel carrier-aggregated modulator structure was proposed. In [16] a fully matched LTE-A carrier aggregation quadplexer based on bulk acoustic wave and surface acoustic wave technologies was proposed. In [17] an efficient carrier aggregation receiver architecture was described,which employed one receive path and a single synthesizer. These works [14]-[17] aimed to study the performance of a single user with CA. Compared with the existing studies, the network performances of multiple users with CA are studied in this work.

Recently, there were some researches on CA for multiple users [18]-[24]. In [17], to improve the performance of the cognitive Ad Hoc networks, based on the proposed Channel-Aggregation Diversity technology the authors proposed two joint power-channel allocation schemes. In [18], to balance the energy efficiency (EE) among multiple users, joint bandwidth and power allocation for both the base station and users was studied. In [19], a joint uplink-downlink algorithm was presented based on a utility function. Although good performance can be achieved with the utilizing methods in [18]-[19], the computation cost was heavy especially for dense small cell networks. In [22], a inter-cell CA technique was proposed to mitigate the non-uniform transmission performance. However, the mitigation of the non-uniform transmission performance was limited. To provide further mitigation against the non-uniform transmission performance, an enhanced inter-cell CA scheme using spread spectrum transmission with variable spreading factor was given in [23]. Note that these methods on CA were all implemented in a centralized manner and we study a distributed game-based approach in this paper.

Besides, many game-based approaches were investigated [25]-[30]. For instance, a pricing game for spatial spectrum sharing-based carrier aggregation was proposed in [27]. In [28], the authors modeled the inter-network CA problem of two networks as a Bayesian game with incomplete information and proposed a distributed algorithm that approaches a neighborhood of the Bayesian NE of the system. But it needed mobile network operators to control the dynamic aggregation of their component carriers. In [29], to alleviate cross-tier and inner-tier interference in heterogeneous networks, a distributed two-dimensional (2D) scheme based on game theory was presented. In [30], the knowledge of the users' positions was combined with Q-learning and game-theoretic approached to enhance the dynamic physical resource allocation for CA in a semi-and uncoordinated deployment of heterogeneous networks. However, for these studies, the different transmission characteristics for the channels in discrete bands was not taken into account. Specifically, for different CA types, i.e., intra-band and inter-band CA, their distinct costs should be considered when aggregating different channels. 
The most related work [6] took into account the cost associated with inter-band CA. CA for autonomous networks operating in shared spectrum was studied and a game-based approach was proposed. However, the defined utility function in [6] was not rational to reflect the throughput, e.g., for player $\mathrm{n}$, if the measured interference in one channel was larger than specified interference threshold, this channel can not be utilized for communication, i.e., n's obtained throughput should be zero rather than a minus value calculated by [6]. In addition, due to the limited transmitting power of base stations, especially for lower power of small cell base stations, the mutual interference between cells should occur in a local area. However, the authors ignored the characteristic of local interference in the design of utility function in [6]. On the contrary, we not only overcome the aforementioned disadvantages, but also define a new utility function to capture the throughput from a different perspective.

\section{System Model and Problem Formulation}

Consider a small cell network with $N$ small cell base stations (SCBSs). For presentation, we use $\mathrm{N}=\{1, \ldots, N\}$ to represent the set of SCBSs. Motivated by [31], assume that SCBSs can exchange information (e.g., location, strategy and utility) through the gateway (See Fig.1). There are $B$ frequency bands available $(B=\{1, \ldots, B\}$ ) in this network area and for each band the number of orthogonal channels available is $K_{b}(1 \leq b \leq B)$. It is assumed that each channel has the same bandwidth and all SCBSs can aggregate different channels in different bands, which means that each SCBS can simultaneously operate on multiple contiguous and non-contiguous channels in the same band or different bands. We denote $M_{i}$ as the maximum number of channels that SCBS $i$ can simultaneously operate on, and $M=\sum_{j=1}^{B} K_{j}$ as the total number of available channels [6]. Therefore, the cardinality of SCBS $i$ 's strategy space is : $\left|\mathrm{A}_{i}\right|=\sum_{n=1}^{M_{i}}\left(\begin{array}{c}M \\ n\end{array}\right)=\sum_{n=1}^{M_{i}} \frac{M(M-1) \cdots(M-n+1)}{n(n-1) \cdots 1}$, where $\mathrm{A}_{i}$ is the set of all available strategy of SCBS $i$.

As is shown in Fig.1, seven SCBSs are deployed in a geographic area randomly. All SCBSs can exchange information with each other through the gateway. Besides, there are three bands available for the network. Using a proper approach, each SCBS decides the aggregated channels in a distributed manner.

According to Shannon equation, when SCBS $i$ communicates in a channel, e.g., $c$, the channel capacity in $c$ can be calculated:

$$
C_{i}(c)=B(c) \times \log _{2}\left(1+\frac{P_{i}(c)}{\sigma^{2}+I_{i}(c)}\right),
$$

where $B(c)$ is the bandwidth of $c . P_{i}(c)$ is the transmitting power of the SCBS in $c . \sigma^{2}$ is the power of the background noise. $I_{i}(c)$ is the interference caused by other cells transmitting in $c$. However, depending on CA technology, the SCBS $i$ may not transmit on only one channel but aggregate more channels for communication. Note that CA is different from the multiple channel selection. When aggregating channels for CA, we must take whether these channels are in different bands and how many bands they cross into account. The reason is that CA with contiguous component carriers needs only one fast Fourier transform (FFT), comparatively CA with non-contiguous component carriers requires separate FFT. In addition, the degree of flexibility in performing CA should also be taken into account. Therefore, in order to present its payoff when SCBS $i$ selects the strategy $a_{i}$, we define the payoff of SCBS $i$ as follows: 


$$
R_{i}\left(a_{i}\right)=\sum_{c_{j} \in a_{i}} C_{i}\left(c_{j}\right) / \Delta-\frac{\left(b\left(a_{i}\right)-1\right)}{b_{M}} \delta,
$$

where $a_{i}$ is the set of channels that SCBS $i$ aggregates. $c_{j}$ is one of channels in the strategy $a_{i}$. $C_{i}\left(c_{j}\right)$ is given by equation(1). $\Delta$ is the normalization factor. $b\left(a_{i}\right)$ is the number of bands that SCBS $i$ accesses when selecting strategy $a_{i}$, and $\left|b\left(a_{i}\right)\right| \leq\left|a_{i}\right| . b_{M}$ is a constant that denotes the maximum number of bands that SCBS $i$ can simultaneously deploy (Fig. 1). $\delta$ is the parameter that each base station can tune to reflect its degree of flexibility in performing CA [6], and smaller $\delta$ represents smaller cost when aggregating channels in different bands.

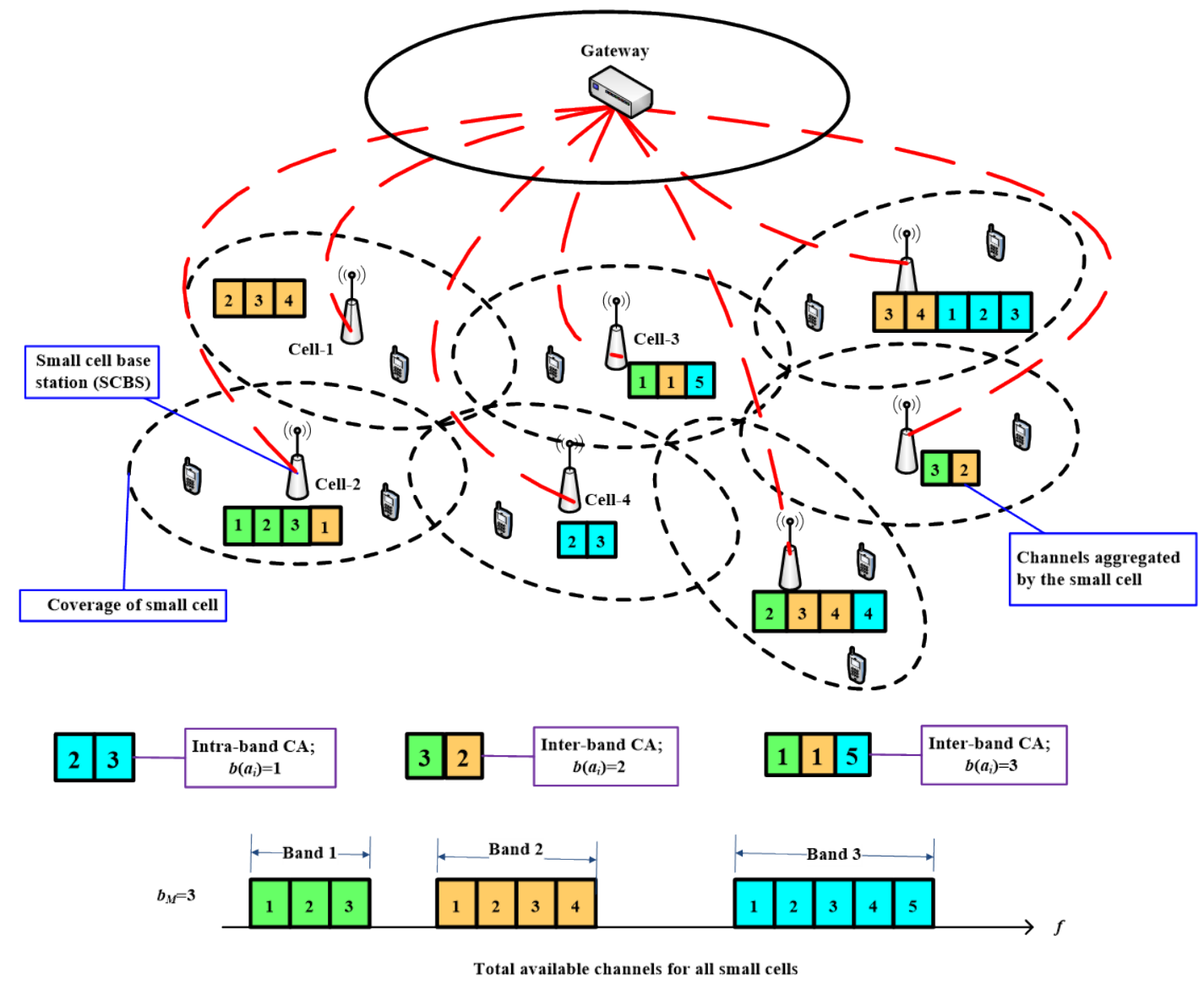

Fig. 1. An example of network model. Different colors represent different frequency bands. Each small rectangle represents a channel. Networks can transmit on multiple channels in the same frequency band (intra-band CA) or in multiple frequency bands (inter-band CA). The red broken lines represent the virtual connection between small cells and the gateway.

For an individual SCBS $i$, the payoff $R_{i}\left(a_{i}\right)$ should be maximized. From a network-centric perspective, the total payoff of all the SCBSs, which is regarded as the network throughput, i.e., $\sum_{i=1}^{N} R_{i}\left(a_{i}\right)$, should be maximized. Therefore, we formulate the problem of CA in cognitive small cell networks as follows: 


$$
\text { P1: } \quad \max \sum_{i=1}^{N} R_{i}\left(a_{i}\right)
$$

According to equation (1), (2) and (3), in order to improve the capacity of the network, we must mitigate mutual interference among the small cells while other parameters stay. Thus, we model the problem $P 1$ as a congest game as follows.

\section{Game Model and Spatial Adaptive Play Algorithm with Heterogeneous Learning Parameters}

\subsection{Game Model}

Due to the fact that small cells have lower transmission power and smaller coverage, a SCBS only interferes with its adjacent ones in a limited range rather than interacts with all SCBSs in a given network [32]. Specifically, if the distance $d_{i j}$ between SCBS $i$ and $j$ is smaller than a predefined threshold $d_{0}$, then they interfere with each other when transmitting on the same channel. That is to say, if the distance $d_{i j}$ between SCBS $i$ and $j$ is larger than a predefined threshold $d_{0}$, they can reuse the spectrum, i.e., transmit on the same channels simultaneously without mutual interference, to improve the spectrum efficiency. For presentation, we denote the neighboring SCBS set of SCBS $i$ as $\mathrm{J}_{i}$, i.e., $\mathrm{J}_{i}=\left\{j \in \mathrm{N}: d_{i j}<d_{0}\right\}$. When two or more neighboring small cells transmit on the same channel, they collide with each other and mutual interference occur, i.e., we only consider co-channel interference. When SCBS $i$ chooses the strategy $a_{i}$ we define corresponding collision level as follows:

$$
f_{i}\left(a_{i}, a_{\mathrm{J}_{i}}\right)=\sum_{c_{j} \in a_{i}} f_{i}\left(c_{j}, a_{\mathrm{J}_{i}}\right),
$$

where $f_{i}\left(c_{j}, a_{\mathrm{J}_{i}}\right)$ is the following indicator function:

$$
f_{i}\left(c_{j}, a_{\mathrm{J}_{i}}\right)=\left|I_{i}\left(c_{j}, a_{\mathrm{J}_{i}}\right)\right|, \quad I_{i}\left(c_{j}, a_{\mathrm{J}_{i}}\right)=\left\{k \in \mathrm{J}_{i}: c_{j} \in a_{k}\right\}
$$

Accordingly, a quantitative characterization of the network collision level is given as follows:

$$
I_{0}=\frac{1}{2} \sum_{i \in \mathrm{N}} f_{i}\left(a_{i}, a_{\mathrm{J}_{i}}\right),
$$

where $a_{j_{i}}$ is the strategy profile of $i$ 's adjacent SCBSs.

It is noted that $f_{i}\left(c_{j}, a_{j_{i}}\right)$ denotes the number of adjacent SCBSs who also select channel $c_{j}$ while $c_{j} \in a_{i}$. For the reason that every small cell prefers aggregating more spectrum resources, a lower collision level and less inter-band cost for better payoff. This motivates us to define the utility function as following:

$$
u_{i}\left(a_{i}, a_{-i}\right)=\left|a_{i}\right|-f_{i}\left(a_{i}, a_{\mathrm{J}_{i}}\right)-\frac{\left(b\left(a_{i}\right)-1\right)}{b_{M}} \delta,
$$

where $a_{-i}$ is the strategy selection profile of all SCBSs except $i .\left|a_{i}\right|$ is the cardinality of the channel set $a_{i} \cdot f_{i}\left(a_{i}, a_{\mathrm{J}_{i}}\right)$ is given by (4). $\left(b\left(a_{i}\right)-1\right) * \delta / b_{M}$ represents the cost of inter-band CA with the same as the last part in (2). If $a_{i}$ crosses only one band this item will be zero, i.e., no cost is taken for CA. If $a_{i}$ crosses two or more bands this item will be greater than zero 
which means that cost is taken for CA. For example, in Fig.1, Cell-2 interfers with Cell-1, Cell-3 and Cell-4. When Cell-2 aggregates the channels shown in Fig.1, $\left|a_{2}\right|=4$, $f_{2}\left(a_{2}, a_{\mathrm{J}_{2}}\right)=2, b\left(a_{i}\right)=2$, the utility of Cell-2 is equal to 1.5 (assume that $\delta=1.5$ ).

Formally, the game model can be denoted as:

$$
\mathrm{G}=\left(\mathrm{N},\left\{\mathrm{A}_{i}\right\}_{i \in \mathrm{N}},\left\{u_{i}\right\}_{i \in \mathrm{N}}\right)
$$

where $\mathrm{N}=\{1, \ldots, N\}$ is a set of players (SCBSs), $\mathrm{A}_{i}$ is the set of player $i$ 's available strategies (set of channels), and $u_{i}$ is the utility function defined by (6). Due to the limited interference range, the utility function can also be expressed as $u_{i}\left(a_{i}, a_{\mathrm{J}_{i}}\right)$, i.e.:

$$
u_{i}\left(a_{i}, a_{-i}\right)=u_{i}\left(a_{i}, a_{\mathrm{J}_{i}}\right)
$$

where $a_{i}$ is the strategy of player $i$ and $a_{\mathrm{J}_{i}}$ is the strategy profile of $i$ 's neighboring players.

Then the proposed channel selection game can be expressed as:

$$
\text { G: } \max _{a_{i} \in \mathrm{A}_{i}} u_{i}\left(a_{i}, a_{\mathrm{J}_{i}}\right)
$$

To analyze the properties of the formulated game, we first present the concept of Nash equilibrium, which is the most well-known stable solution for non-cooperative game model.

Definition 1(NE). A strategy selection profile $a^{*}=\left(a_{1}^{*}, \ldots, a_{N}^{*}\right)$ is a pure strategy NE if and only if no player can improve its utility by deviating unilaterally, i.e.,

$$
u_{i}\left(a_{i}^{*}, a_{-i}^{*}\right) \geq u_{i}\left(a_{i}, a_{-i}^{*}\right), \quad \forall i \in \mathrm{N}, \forall a_{i} \in \mathrm{A}_{i}, a_{i} \neq a_{i}^{*}
$$

Definition 2 (Exact potential game [33]). A game is an exact potential game if there exists an ordinal potential function $\Phi: \mathrm{A}_{1} \times \cdots \times \mathrm{A}_{N} \rightarrow R$ such that for all $i \in \mathrm{N}$, all $a_{i} \in \mathrm{A}_{i}$ and $a_{i}^{\prime} \in \mathrm{A}_{i}$, the following holds:

$$
u_{i}\left(a_{i}, a_{-i}\right)-u_{i}\left(a_{i}^{\prime}, a_{-i}\right)=\Phi\left(a_{i}, a_{-i}\right)-\Phi\left(a_{i}^{\prime}, a_{-i}\right)
$$

i.e., the change in the utility function caused by the unilateral action change of an arbitrary player is exactly the same with that in the potential function.

The given definitions are straightforwardly obtained from game theory [33]. The properties of the proposed game are characterized by the following theorem.

Theorem 1. $G$ is an exact potential game which has at least one pure strategy NE.

Proof: In order to prove $\mathrm{G}$ to be an exact potential game, we construct a potential function as follows:

$$
\Phi\left(a_{i}, a_{-i}\right)=\sum_{i=1}^{N}\left|a_{i}\right|-\frac{1}{2} \sum_{i=1}^{N} f_{i}\left(a_{i}, a_{\mathrm{J}_{i}}\right)-\sum_{i=1}^{N} \frac{\left(b\left(a_{i}\right)-1\right)}{b_{M}} \delta
$$

If player $i$ changes its selected strategy unilaterally from $a_{i}=\left\{c_{1}, c_{2}, \ldots, c_{h}\right\}$ to $a_{i}^{*}=\left\{c_{1}^{*}, c_{2}^{*}, \ldots, c_{H}^{*}\right\}$, the amount of change in its utility function is given by: 


$$
\begin{aligned}
& u_{i}\left(a_{i}^{*}, a_{-i}\right)-u_{i}\left(a_{i}, a_{-i}\right) \\
& =u_{i}\left(a_{i}^{*}, a_{\jmath_{i}}\right)-u_{i}\left(a_{i}, a_{\mathrm{J}_{i}}\right) \\
& =\left|a_{i}^{*}\right|-\left|a_{i}\right|+f_{i}\left(a_{i}, a_{\mathrm{J}_{i}}\right)-f_{i}\left(a_{i}^{*}, a_{\mathrm{J}_{i}}\right)+\frac{\left(b\left(a_{i}\right)-1\right)}{b_{M}} \delta-\frac{\left(b\left(a_{i}^{*}\right)-1\right)}{b_{M}} \delta \\
& =\left|a_{i}^{*}\right|-\left|a_{i}\right|+\sum_{c_{j} \in a_{i}} f_{i}\left(c_{j}, a_{\mathrm{J}_{i}}\right)-\sum_{c_{j} \in a_{i}^{*}} f_{i}\left(c_{j}, a_{\mathrm{J}_{i}}\right)+\frac{\left(b\left(a_{i}\right)-1\right)}{b_{M}} \delta-\frac{\left(b\left(a_{i}^{*}\right)-1\right)}{b_{M}} \delta
\end{aligned}
$$

Accordingly, the amount of change in the potential function caused by the unilateral change $\left(a_{i} \rightarrow a_{i}^{*}\right)$ is given by:

$$
\begin{aligned}
& \Phi\left(a_{i}^{*}, a_{-i}\right)-\Phi\left(a_{i}, a_{-i}\right) \\
& =\sum_{i=1}^{N}\left|a_{i}^{*}\right|-\sum_{i=1}^{N}\left|a_{i}\right|+\frac{1}{2} \sum_{i=1}^{N} f_{i}\left(a_{i}, a_{\mathrm{J}_{i}}\right)-\frac{1}{2} \sum_{i=1}^{N} f_{i}\left(a_{i}^{*}, a_{\mathrm{J}_{i}}\right)+\sum_{i=1}^{N} \frac{\left(b\left(a_{i}\right)-1\right)}{b_{M}} \delta-\sum_{i=1}^{N} \frac{\left(b\left(a_{i}^{*}\right)-1\right)}{b_{M}} \delta \\
& =\left|a_{i}^{*}\right|-\left|a_{i}\right|+\frac{1}{2} \sum_{i=1}^{N}\left[\sum_{c j \in a_{i}} f_{i}\left(c_{j}, a_{\mathrm{J}_{i}}\right)-\sum_{\substack{c_{j}^{*} \in a_{i}^{*} \\
\text { in }}} f_{i}\left(c_{j}^{*}, a_{\mathrm{J}_{i}}\right)\right]+\frac{\left(b\left(a_{i}\right)-1\right)}{b_{M}} \delta-\frac{\left(b\left(a_{i}^{*}\right)-1\right)}{b_{M}} \delta
\end{aligned}
$$

Compare (14) with (15), we know that proving (12) is equal to proving the following expression:

$$
\frac{1}{2} \sum_{i=1}^{N}\left[\sum_{c_{j} \in a_{i}} f\left(c_{j}, a_{\mathrm{J}_{i}}\right)-\sum_{c_{j}^{*} \in a_{i}^{*}} f\left(c_{j}^{*}, a_{\mathrm{J}_{i}}\right)\right]=\sum_{c_{j} \in a_{i}} f\left(c_{j}, a_{\mathrm{J}_{i}}\right)-\sum_{c_{j}^{*} \in a_{i}^{*}} f\left(c_{j}^{*}, a_{\mathrm{J}_{i}}\right)
$$

For presentation, without loss of generality the change $a_{i} \rightarrow a_{i}^{*}$ can be seen as the change of corresponding channels, i.e., $\left\{c_{j} \rightarrow c_{j}^{*}, c_{j} \in a_{i}, c_{j}^{*} \in a_{i}^{*}\right\}$.

Therefore,

$$
\begin{aligned}
& \sum_{i=1}^{N}\left[\sum_{c_{j} \in a_{i}} f\left(c_{j}, a_{\mathrm{J}_{i}}\right)-\sum_{c_{j}^{*} \in a_{i}^{*}} f\left(c_{j}^{*}, a_{\mathrm{J}_{i}}\right)\right] \\
& =\sum_{c_{j} \in a_{i}} f\left(c_{j}, a_{\mathrm{J}_{i}}\right)-\sum_{c_{j}^{*} \in a_{i}^{*}} f\left(c_{j}^{*}, a_{\mathrm{J}_{i}}\right)+\sum_{k \in \mathrm{J}_{i}}\left[\sum_{c_{k} \in a_{k}} f\left(c_{k}, a_{\mathrm{J}_{i}}\right)-\sum_{c_{k} \in a_{k}} f\left(c_{k}, a_{\mathrm{J}_{i}}^{*}\right)\right] \\
& =\sum_{c_{j} \in a_{i}} f\left(c_{j}, a_{\mathrm{J}_{i}}\right)-\sum_{c_{j}^{*} \in a_{i}^{*}} f\left(c_{j}^{*}, a_{\mathrm{J}_{i}}\right)+\sum_{c_{j} \rightarrow c_{j}^{*}, c_{k} \in a_{k}} \sum_{k \in \mathrm{J}_{i}}\left[f\left(c_{k}, a_{\mathrm{J}_{k}}\right)-f\left(c_{k}, a_{\mathrm{J}_{k}}^{*}\right)\right] \\
& =\sum_{c_{j} \in a_{i}} f\left(c_{j}, a_{\mathrm{J}_{i}}\right)-\sum_{c_{j}^{*} \in a_{i}^{*}} f\left(c_{j}^{*}, a_{\mathrm{J}_{i}}\right)+\sum_{k \in \mathrm{J}_{i}} \sum_{c_{j} \rightarrow c_{j}^{*}, c_{k} \in a_{k}}\left[f\left(c_{k}, a_{\mathrm{J}_{k}}\right)-f\left(c_{k}, a_{\mathrm{J}_{k}}^{*}\right)\right] \\
& =\sum_{c_{j} \in a_{i}} f\left(c_{j}, a_{\mathrm{J}_{i}}\right)-\sum_{c_{j}^{*} \in a_{i}^{*}} f\left(c_{j}^{*}, a_{\mathrm{J}_{i}}\right)+\sum_{k \in I_{i}\left(c_{j}, a_{\mathrm{J}_{i}}\right)} c_{c_{j} \rightarrow c_{j}^{*}, c_{k} \in a_{k}}\left[f\left(c_{k}, a_{\mathrm{J}_{k}}\right)-f\left(c_{k}, a_{\mathrm{J}_{k}}^{*}\right)\right] \\
& +\sum_{k \in I_{i}\left(c_{j}^{*}, a_{\mathrm{J}_{i}}\right)} c_{c_{j} \rightarrow c_{j}^{*}, c_{k} \in a_{k}}\left[f\left(c_{k}, a_{\mathrm{J}_{k}}\right)-f\left(c_{k}, a_{\mathrm{J}_{k}}^{*}\right)\right]+\sum_{k \in K, k \neq i} \sum_{c_{j} \rightarrow c_{j}^{*}, c_{k} \in a_{k}}\left[f\left(c_{k}, a_{\mathrm{J}_{k}}\right)-f\left(c_{k}, a_{\mathrm{J}_{k}}^{*}\right)\right]
\end{aligned}
$$

where $\kappa=\mathrm{J}_{i} \backslash\left\{I_{i}\left(c_{j}, a_{\mathrm{J}_{i}}\right) \cup I_{i}\left(c_{j}^{*}, a_{\mathrm{J}_{i}}\right)\right\}$, and $A \backslash B$ means to delete the set $B$ from the set $A$. $f\left(c_{k}, a_{J_{k}}^{*}\right)$ is the collision level of player $k$ in channel $c_{k}$ after player $i$ unilaterally changes 
its strategy. Due to the strategy change of player $i$ only impacts its neighboring player, following results can be obtained:

$$
\begin{cases}f\left(c_{k}, a_{\mathrm{J}_{k}}\right)-f\left(c_{k}, a_{\mathrm{J}_{k}}^{*}\right)=1, & \forall k \in I_{i}\left(c_{j}, a_{\mathrm{J}_{i}}\right) \\ f\left(c_{k}, a_{\mathrm{J}_{k}}\right)-f\left(c_{k}, a_{\mathrm{J}_{k}}^{*}\right)=1, & \forall k \in I_{i}\left(c_{j}^{*}, a_{\mathrm{J}_{i}}\right) \\ f\left(c_{k}, a_{\mathrm{J}_{k}}\right)-f\left(c_{k}, a_{\mathrm{J}_{k}}^{*}\right)=0, & \forall k \in \kappa, k \neq i\end{cases}
$$

Then, according to (18) we can derive:

$$
\begin{aligned}
& \sum_{i=1}^{N}\left[\sum_{c_{j} \in a_{i}} f\left(c_{j}, a_{\mathrm{J}_{i}}\right)-\sum_{c_{j}^{*} \in a_{i}^{*}} f\left(c_{j}^{*}, a_{\mathrm{J}_{i}}\right)\right] \\
& =\sum_{c_{j} \in a_{i}} f\left(c_{j}, a_{\mathrm{J}_{i}}\right)-\sum_{c_{j}^{*} \in a_{i}^{*}} f\left(c_{j}^{*}, a_{\mathrm{J}_{i}}\right)+\sum_{c_{j} \in a_{i}} f\left(c_{j}, a_{\mathrm{J}_{i}}\right)-\sum_{c_{j}^{*} \in a_{i}^{*}} f\left(c_{j}^{*}, a_{\mathrm{J}_{i}}\right) \\
& =2\left[\sum_{c_{j} \in a_{i}} f\left(c_{j}, a_{\mathrm{J}_{i}}\right)-\sum_{c_{j}^{*} \in a_{i}^{*}} f\left(c_{j}^{*}, a_{\mathrm{J}_{i}}\right)\right]
\end{aligned}
$$

Thus, (16) is derived and the following holds:

$$
u_{i}\left(a_{i}^{*}, a_{-i}\right)-u_{i}\left(a_{i}, a_{-i}\right)=\Phi\left(a_{i}^{*}, a_{-i}\right)-\Phi\left(a_{i}, a_{-i}\right)
$$

i.e., when an arbitrary player changes its selected strategy unilaterally, the change in the individual utility function caused by any player's unilateral deviation is the same as the change in the potential function. According to Definition 2, G is an exact potential game with network utility serving as the potential function.

Exact potential game belongs to potential games, which have been widely applied to wireless communication systems. Potential game exhibits several nice properties and the most important two are as follows [32]-[33].

1) Every potential game has at least one pure strategy NE.

2) Any global or local maxima of the potential function constitutes a pure strategy NE.

Based on the first property, theorem 1 is proved.

We note that this potential function (13) has strong relevance with the network utility according to (6) and (7). Moreover, the utility function (7) is bound up with the payoff level. The reason is that higher collision level causes the utility smaller as well as the payoff level. Therefore, the network utility can reflect the level of total network throughput, so can the potential function. We conclude that the strategy profile corresponding to the global or local maxima of the potential function can make the network throughput better.

\subsection{Spatial Adaptive Play Algorithm with Heterogeneous Learning Parameters}

Because the distributed CA problem has been now formulated as an exact potential game, the spatial adaptive play (SAP) algorithm [34]-[36] can be applied to achieve a pure NE of the game that maximizes the potential function with arbitrarily high probability. However, it has been shown that the convergence speed of conventional SAP algorithm is slow [32].

The learning parameter $\beta$ of SAP balances the tradeoff between exploration and exploitation. Smaller implies that the CR users are more willing to choose an suboptimal action to explore, whereas higher implies that they are prone to choose the best response action. Motivated by the idea proposed in [37], we introduce a heterogeneous learning parameter, i.e., $\beta\left|\mathrm{J}_{i}\right| / \min _{n \in \mathbb{N}}\left(\left|\mathrm{J}_{n}\right|\right)$, where $\left|\mathrm{J}_{i}\right|$ is the number of small cell $i$ 's neighboring cells, to balance the 
tradeoff between exploration and exploitation and accelerate the converging speed. The reason is that small cells are usually deployed randomly, the density of small cells is distinct in different local areas. For a given small cell network, $\min _{n \in \mathbb{N}}\left(\left|\mathrm{J}_{n}\right|\right)$ is a constant, and $\left|\mathrm{J}_{i}\right|$ is larger for the denser area. Note that $\beta\left|\mathrm{J}_{i}\right| / \min _{n \in \mathbb{N}}\left(\left|\mathrm{J}_{n}\right|\right)$ is larger than the homogeneous learning parameter $\beta$ and in the denser area $\beta\left|\mathrm{J}_{i}\right| / \min _{n \in \mathbb{N}}\left(\left|\mathrm{J}_{n}\right|\right)$ is larger for a fixed $\beta$. During the learning period, players (small cells) with the larger learning parameter $\beta\left|\mathrm{J}_{i}\right| / \min _{n \in \mathrm{N}}\left(\left|\mathrm{J}_{n}\right|\right)$ will have the higher probability (defined by (21)) to choose better actions. Thus, accelerate the process of choosing the optimal actions for every players, i.e., improve the converging speed.

Therefore, we propose a spatial adaptive play algorithm with heterogeneous learning parameters (SAP-H). The specific algorithm is described in Algorithm 1.

Algorithm 1. Spatial adaptive play algorithm with heterogeneous learning parameters $(\mathrm{SAP}-\mathrm{H})$.

Initialization: Set the iteration index $k=0$, let each player $n, \forall n \in N$, randomly select an available strategy $a_{n}(0)$ from the set of its available strategies with equal probability.

Step 1: All players exchanges information with their adjacent players through the gateway.

Step 2: Select a player $i$ randomly.

Step 3: Player $i$ calculates the utility functions over its all available strategies, i.e., $u_{i}\left(\bar{a}_{i}, a_{J_{i}}(k)\right), \forall \bar{a}_{i} \in \mathrm{A}_{i}$, while all other players repeat their selections, i.e., $a_{-i}(k+1)=a_{-i}(k)$. Then, player $i$ updates its mixed strategy:

$$
q_{i}^{a_{i}}(k+1)=\frac{\exp \left\{\left[\left|\mathrm{J}_{i}\right| / \min _{n \in \mathrm{N}}\left(\left|\mathrm{J}_{n}\right|\right)\right] \beta u_{i}\left(a_{i}, a_{J_{i}}(k)\right)\right\}}{\sum_{\bar{a}_{i} \in \mathrm{A}_{i}} \exp \left\{\left[\left|\mathrm{J}_{i}\right| / \min _{n \in \mathrm{N}}\left(\left|\mathrm{J}_{n}\right|\right)\right] \beta u_{i}\left(\bar{a}_{i}, a_{J_{i}}(k)\right)\right\}},
$$

where $q_{i}^{a_{i}}(k+1)$ is the probability when play $i$ select the strategy $a_{i}$ at iteration $k+1$. $\beta=1+5 k / 1000 ;\left|\mathrm{J}_{i}\right|$ is the number of neighboring player of player $i ;\left|\mathrm{J}_{i}\right| / \min _{n \in N}\left(\left|\mathrm{~J}_{n}\right|\right)$ is a dynamic factor which can vary according to the density of small cells deployed. Player $i$ selects a new strategy $a_{n}(k+1)$ according to the mixed strategy $q_{n}(k+1)$.

Step 4: If $k$ exceeds predefined maximal number of learning, end; otherwise return to Step 2.

Theorem 2. In a potential game in which all players adhere to SAP-H, the unique stationary distribution $\mu(\mathrm{a}) \in \Delta(\mathrm{A})$ of the joint action profiles, $\forall \beta>0$, is given as

$$
\mu(\mathrm{a})=\frac{\exp \left\{\left[\left|\mathrm{J}_{i}\right| / \min _{n \in \mathbb{N}}\left(\left|\mathrm{J}_{n}\right|\right)\right] \beta \Phi(a)\right\}}{\sum_{s \in \mathrm{A}} \exp \left\{\left[\left|\mathrm{J}_{i}\right| / \min _{n \in \mathbb{N}}\left(\left|\mathrm{J}_{n}\right|\right)\right] \beta \Phi(s)\right\}},
$$

where $\Phi()$ is the potential function of the games specified by (13), A is the set of available selection profiles of all players, i.e., $A=A_{1} \otimes A_{2} \otimes \cdots \otimes A_{N}$.

Proof: The following proof follows the proof lines given in [32] [34]. 
In the considered network scenario, once the network topology is fixed, $\left|J_{i}\right| / \min _{n \in \mathbb{N}}\left(\left|J_{n}\right|\right)$ is a constant for a fixed SCBS i but distinct for different SCBSs. Consequently, it is rational to replace $\left[\left|\mathrm{J}_{i}\right| / \min _{n \in \mathrm{N}}\left(\left|\mathrm{J}_{n}\right|\right)\right] \beta$ with $\beta_{i}$. Thus, (22) can be written as:

$$
\mu(\mathrm{a})=\frac{\exp \left\{\beta_{i} \Phi(a)\right\}}{\sum_{s \in \mathrm{A}} \exp \left\{\beta_{i} \Phi(s)\right\}}
$$

Denote the action selection profile at the $k$ th iteration by $a(k)=\left\{a_{1}(k), \ldots, a_{N}(k)\right\}$, where $a_{n}(k)$ is the action of player $n$. Note that an action selection profile corresponds to a network state. Because the network state $a(k+1)$ just depends on $a(k)$ rather than the network states before the $k$ th iteration. Therefore, we can interpret the learning process as a discrete time Markov process, which is irreducible and aperiodic. According to the nature of the discrete time Markov process, an unique stationary distribution can be found.

In order to show that (23) is the mentioned unique stationary distribution, we can verify that (23) deduces to the balanced equations of the Markov process. The details are as follows:

Denote any two arbitrary neighboring network state by $a$ and $b, a, b \in \mathrm{A}$, and the transmission probability from $a$ to $b$, by $P_{a b}=\operatorname{Pr}[a(k+1)=b \mid a(k)=a]$. Since in one iteration only exactly one node is selected to update its selection in the proposed algorithm, there is at most one element that can be changed in the network states between any two successive iterations. Thus, there are only two nontrivial cases: 1) $a$ and $b$ differ by exactly one element, or 2) $a=b$.

For case 1), specifically, we suppose that $a$ and $b$ differ by the $i$ th element. Since node has a probability $1 / N$ of being chosen in any given iteration, it follows that

$$
\mu(\mathrm{a}) P_{a b}=\left(\frac{1}{N}\right) \times\left(\frac{\exp \left\{\beta_{i} \Phi(a)\right\}_{i \in \mathrm{N}}}{\sum_{s \in \mathrm{A}} \exp \left\{\beta_{i} \Phi(s)\right\}_{i \in \mathrm{N}}}\right) \times P_{a b},
$$

where the the iteration index $k$ is omitted and that has no effect.

Note that

$$
P_{a b}=\left(\frac{\exp \left\{\beta_{i} u\left(b_{i}, b_{-i}(k)\right)\right\}_{i \in \mathrm{N}}}{\sum_{\bar{b}_{i} \in A_{i}} \exp \left\{\beta_{i} u\left(\bar{b}_{i}, b_{-i}(k)\right)\right\}_{i \in \mathrm{N}}}\right),
$$

where $u\left(b_{i}, b_{-i}(k)\right)$ is the utility function of node $i$ under the network state $b$ at the $k$ th iteration.

Thus,

$$
\mu(\mathrm{a}) P_{a b}=\left(\frac{1}{N}\right) \times\left(\frac{\exp \left\{\beta_{i} \Phi(a)\right\}_{i \in \mathrm{N}}}{\sum_{s \in \mathrm{A}} \exp \left\{\beta_{i} \Phi(s)\right\}_{i \in \mathrm{N}}}\right) \times\left(\frac{\exp \left\{\beta_{i} u\left(b_{i}, b_{-i}(k)\right)\right\}_{i \in \mathrm{N}}}{\sum_{\bar{b}_{i} \in \mathrm{A}_{i}} \exp \left\{\beta_{i} u\left(\bar{b}_{i}, b_{-i}(k)\right)\right\}_{i \in \mathrm{N}}}\right)
$$

Letting

$$
\begin{aligned}
& \lambda=\frac{1 / N}{\left(\sum_{s \in \mathrm{A}} \exp \left\{\beta_{i} \Phi(s)\right\}_{i \in \mathrm{N}}\right) \times\left(\sum_{\bar{b}_{i} \in \mathrm{A}_{i}} \exp \left\{\beta_{i} u_{i}\left(\bar{b}_{i}, b_{-i}\right)\right\}_{i \in \mathrm{N}}\right)} \\
& =\frac{1 / N}{\left(\sum_{s \in \mathrm{A}} \exp \left\{\beta_{i} \Phi(s)\right\}_{i \in \mathrm{N}}\right) \times\left(\sum_{\bar{a}_{i} \in \mathrm{A}_{i}} \exp \left\{\beta_{i} u_{i}\left(\bar{a}_{i}, a_{-i}\right)\right\}_{i \in \mathrm{N}}\right)}
\end{aligned}
$$


According to the fact that the network states $a$ and $b$ differ by the $i$ th element, i.e., $a_{-i}(k) \equiv b_{-i}(k)$, the following equation can be obtained:

$$
\mu(a) P_{a b}=\lambda \exp \left\{\beta_{i} \Phi(a)+\beta_{i} u_{i}\left(b_{i}, b_{-i}\right)\right\}_{i \in \mathrm{N}}
$$

Due to symmetry,

$$
\mu(b) P_{b a}=\lambda \exp \left\{\beta_{i} \Phi(b)+\beta_{i} u_{i}\left(a_{i}, a_{-i}\right)\right\}_{i \in \mathbb{N}}
$$

Therefore,

$$
\frac{\mu(a) P_{a b}}{\mu(b) P_{b a}}=\exp \left\{\beta_{i}[\Phi(a)-\Phi(b)]+\beta_{i}\left[u_{i}\left(b_{i}, b_{-i}\right)-u_{i}\left(a_{i}, a_{-i}\right)\right]\right\}_{i \in \mathrm{N}}
$$

Using (12) in Theorem 1, we obtain

$$
\mu(a) P_{a b}=\mu(b) P_{b a}
$$

For case 2), i.e., $a=b$, (31) naturally holds.

Thus,

$$
\sum_{a \in \mathrm{A}} \mu(a) P_{a b}=\sum_{a \in \mathrm{A}} \mu(b) P_{b a}=\mu(b) \sum_{a \in \mathrm{A}} P_{b a}=\mu(b),
$$

which is exactly the balanced stationary equation of the Markov process. Since SAP-H has unique distribution and the distribution (22) satisfies the balanced equations of its Markov process, we conclude that its stationary distribution must be (22).

Therefore, Theorem 2 is proved.

Theorem 3: With a sufficiently large $\beta$, SAP-H achieves the maximum potential function values with an arbitrarily high probability, where the potential function is defined by (13).

Proof: Theorem 4 in [32] had proved that with a sufficiently large $\beta$, SAP could achieve the maximum potential function value of the game with an arbitrarily high probability. Similarly, as mentioned in the proof of Theorem 2, we replace $\left[\left|\mathrm{J}_{i}\right| / \min _{n \in \mathbb{N}}\left(\left|\mathrm{J}_{n}\right|\right)\right] \beta$ with $\beta_{i}$. Then, (21) can be rewritten as:

$$
q_{i}^{a_{i}}(k+1)=\frac{\exp \left\{\beta_{i} u_{i}\left(a_{i}, a_{J_{i}}(k)\right)\right\}}{\sum_{\bar{a}_{i} \in \mathrm{A}_{i}} \exp \left\{\beta_{i} u_{i}\left(\bar{a}_{i}, a_{J_{i}}(k)\right)\right\}},
$$

which is similar to (30) in [32]. In addition, $\beta \rightarrow \infty$ is equal to $\beta_{i} \rightarrow \infty$ due to the limited value of $\left|\mathrm{J}_{i}\right| / \min _{n \in \mathrm{N}}\left(\left|\mathrm{J}_{n}\right|\right)$, and the updating processes of SAP-H and SAP are the same. Thus, we deduce that the two algorithms have the same converging result, i.e., with a sufficiently large $\beta$, SAP-H also achieves the maximum potential function value of the game with an arbitrarily high probability.

Therefore, Theorem 3 is proved.

For the proposed game, even though the optimal strategy profile may oscillate [36], the maximum potential function value can be achieved with arbitrarily high probability with a sufficiently large $\beta$. Then, according to the strong relevance between the potential function and the network throughput, as specified by Theorem 3, the strategy profile of achieving the optimal potential function can make the network throughput better. Thus, it is a desired learning algorithm because a better solution for problem P1 are achieved via just local information exchange between neighbors. 


\section{Simulation Results and Discussion}

In the following simulation study, we consider a homogeneous small cell network that all SCBSs are randomly located in a $200 \mathrm{~m} \times 200 \mathrm{~m}$ square region. The direct connectivity between the core network and the SAP is maintained by the small-cell gateway. Together with signaling protocol and channel conversions, the small-cell gateway aggregates and integrates traffic from a large number of small cells into the existing mobile networks [8]. In addition, the small-cell gateway can allow the information exchanging among small cells. For the convenience of simulation and presentation, we assume that each small cell has the same transmitting power and the same coverage ${ }^{1}$. An example of the simulated random topology is given by Fig. 2. The coverage distance of each small cell is $20 \mathrm{~m}$, and the interference distance of co-channel communications is set to $60 \mathrm{~m}$. The transmitting power of each small cell is $40 \mathrm{~mW}$. We assume that in the network there are several different bands available, and the transmitting characteritic for different channels in different bands are same. All the channels have the same bandwidth $5 \mathrm{MHz}$. The background noise is $-174 \mathrm{dbm} / \mathrm{Hz}$. The path loss is proportional to square of the transmitting distance.

\subsection{Comparison of Convergence Speed}

For the random network topology which is shown in Fig. 2, there are 20 SCBSs deployed. We consider that there are two frequency bands, i.e., $B=2$, that can be used dynamically and each band has three channels [6], i.e., $K_{b}=3$. Therefore, there are six channels available in the network. The maximum number of channels that each SCBS can simultaneously operate on is three, i.e., $M_{i}=3$. The maximum number of bands that each SCBS can simultaneously deploy is two, i.e., $b_{M}=2$.

On one hand, in Fig. 3 we compare the convergence speed of the proposed SAP-H and the conventional SAP in different network scenarios. The results are obtained by taking 1000 independent trials and then taking expectation for a network topology. It is noted that in the network with 20 small cells the proposed SAP-H converges after about 400 iterations while the conventional SAP does after 900 iterations, and in the network with 40 small cells the proposed SAP-H converges after about 600 iterations while the conventional SAP does after 950 iterations. Therefore, the iterations needed for converging of the proposed SAP-H algorithm is significantly decreased compared with the conventional SAP algorithm.

\footnotetext{
${ }^{1}$ However, it should be emphasized that the proposed game-theoretic solution is general and can be applied to scenarios with heterogeneous converge.
} 


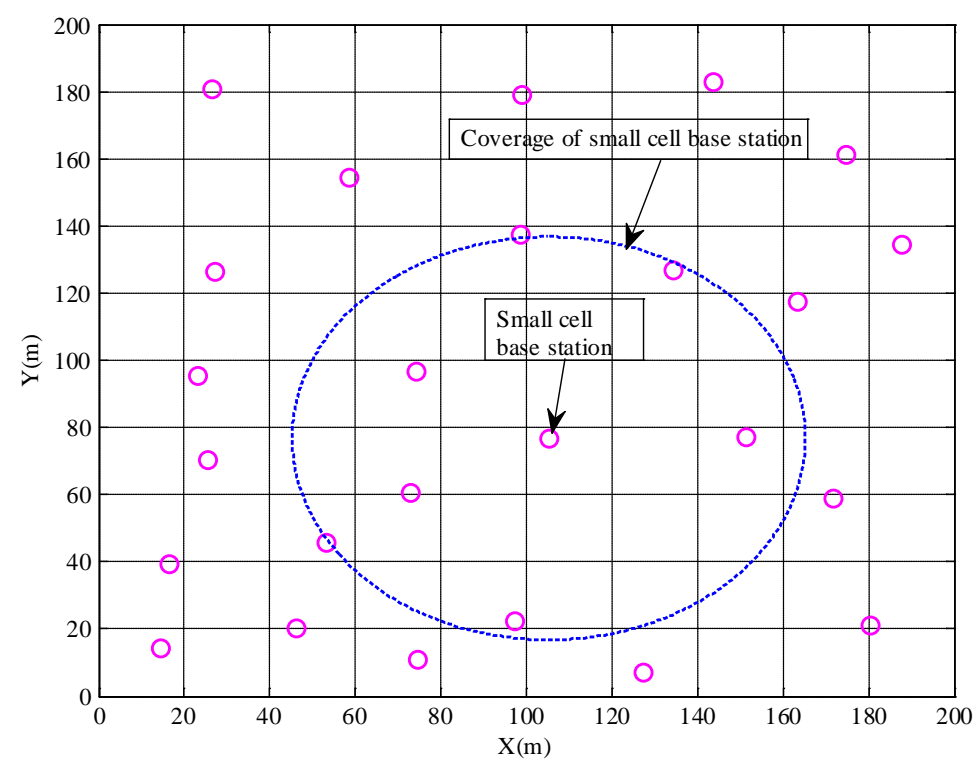

Fig. 2. An example of simulated random network topology with 20 SCBSs. (The small solid circles represent the SCBSs while the large dashed circles represent the region of co-channel interference.)

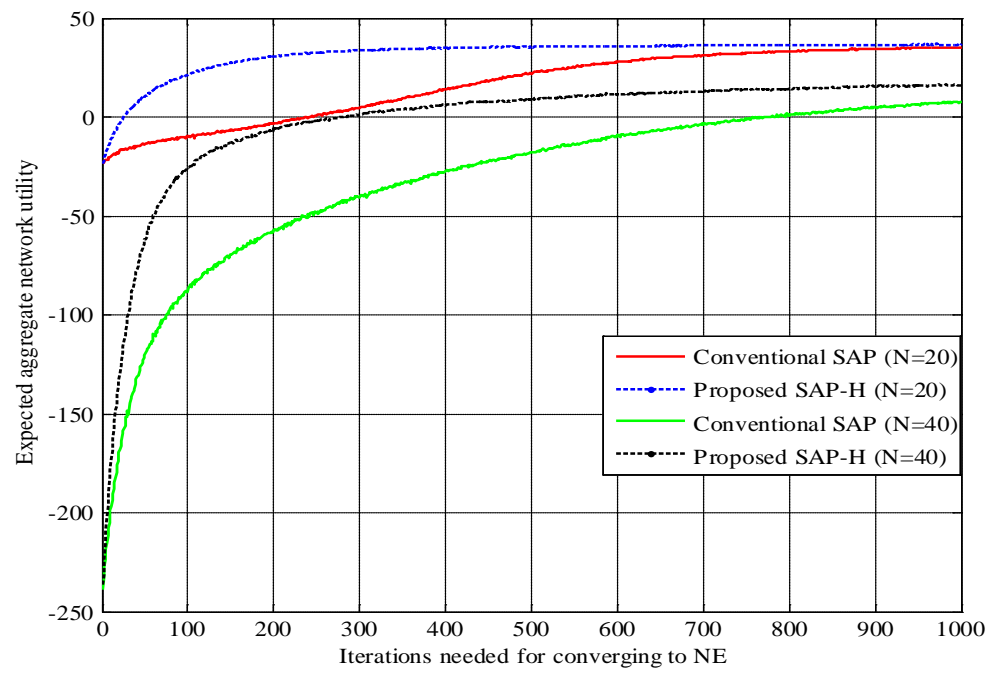

Fig. 3. Comparison of the convergence speed between the conventional SAP and the proposed SAP-H ( $\mathrm{N}$ represents the number of SCBSs in the considered network).

On the other hand, in Fig. 4, we compare the relevance among the network utility, the potential function and the network throughput. It is noted that as the potential function increases, the network utility and the network throughput increases. This result confirms that the relevance among the network utility, the potential function and the network throughput is robust. It is further validated that the strategy profile of achieving the optimal potential function can also make the network utility and the network throughput optimal. Besides, our defined utility function is rational, which can reflect the network capacity. 


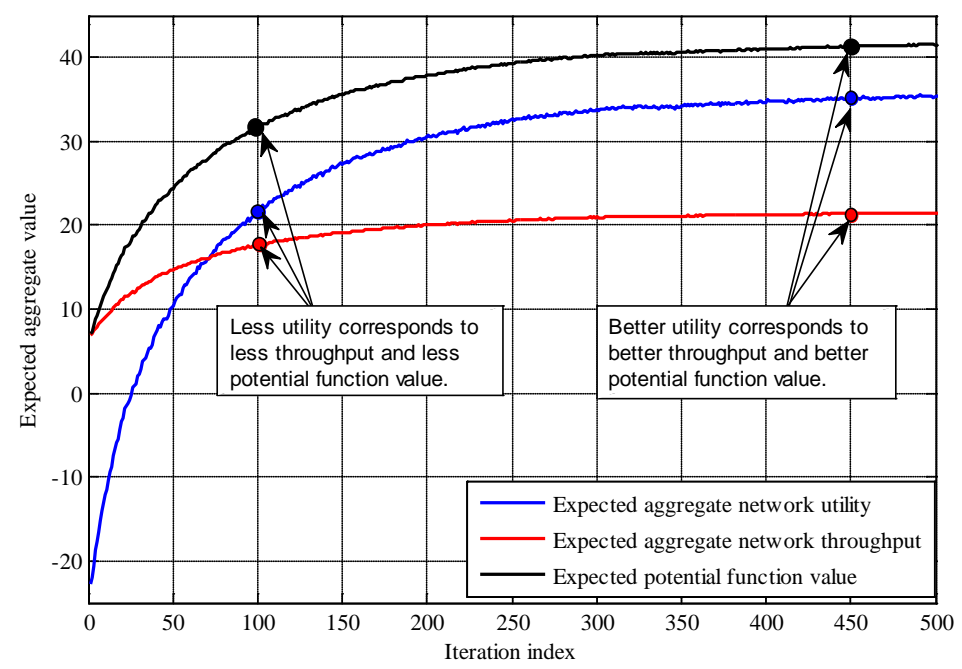

Fig. 4. The relevance among the network utility, the potentialfunction and the network throughput.

\subsection{Throughput Performance}

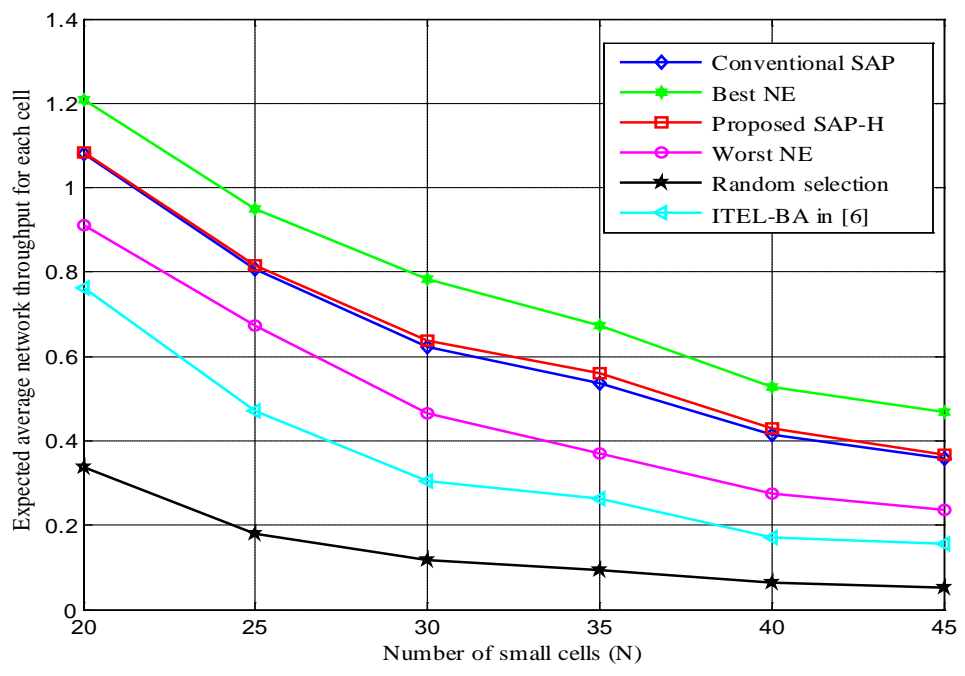

Fig. 5. The expected average throughput for each small cell when varying the number of small cells

$$
\left(B=2, K_{b}=3, M_{i}=3, b_{M}=2\right) \text {. }
$$

The average throughput for each small cell when varying the number of small cells is shown in Fig. 5. It is noted that as the number of small cells increases, i.e., the network density deployed increases, the average throughput for each cell decreases and the tendencies are almost the same for different algorithms. We obtain the best $\mathrm{NE}$ and the worst NE by taking 1000 independent trials and then taking the maximum and minimum throughput after convergence for different network layouts, respectively. Note that the performance of the proposed SAP-H algorithm is close to the best NE and has approximate 10\% improvement than the worst NE. The gap among them is nearly uniform. Also, the several learning 
algorithms significantly outperform the random selection methods. Particularly, SAP-H has a great improvement in terms of the throughput than random selection methods especially for the networks deployed with sparse cells, e.g., 20 small cells. More importantly, compared to conventional SAP, its performance have a little improvement. In addition, the performance of our proposed SAP-H has about 25\% improvement than the ITEL-BA algorithm proposed in [6]. The reason is that the defined utility function in [6] was not rational to reflect the throughput, e.g., for player $n$, if measured interference in one channel was larger than specified interference threshold, this channel could not be utilized for communication, i.e., n's obtained throughput should be zero rather than a minus value calculated by [6].

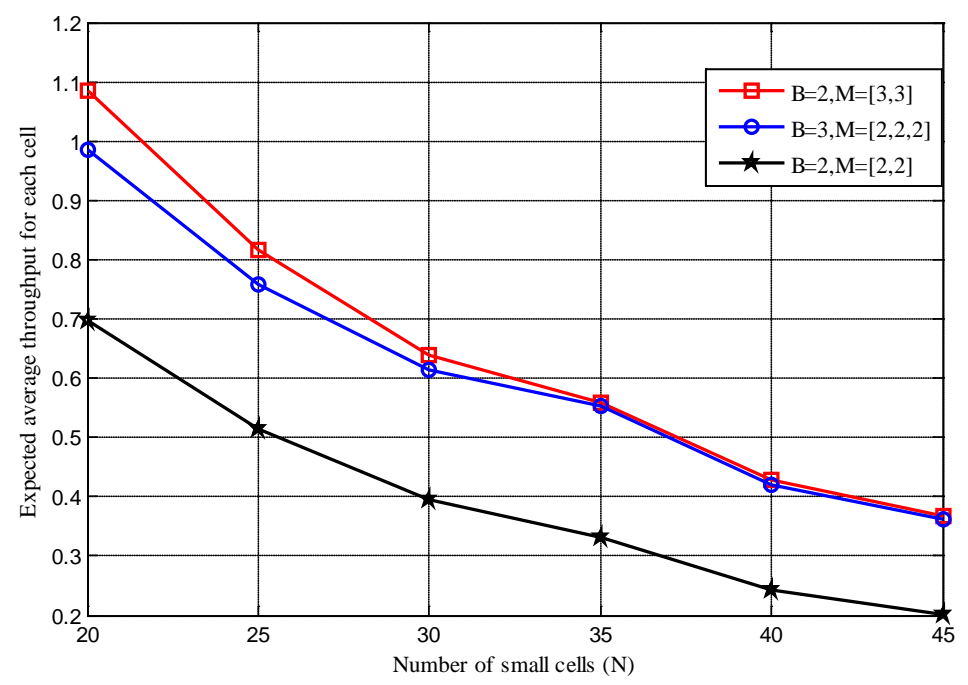

Fig. 6. The comparison of expected average throughput for each small cell with different spectrum resource available ( $B$ denotes the number of bands available, $M$ shows the number of channels in each band).

In order to study the impact on the network performance with different spectrum available, we compare the average throughput with different spectrum resources available in Fig. 6. For $M=[3,3]$ and $M=[2,2,2]$, there are both 6 channels available. It is noted that in a network with 20 cells the throughput for $M=[3,3]$ has $10 \%$ improvement than $M=[2,2,2]$. That is to say, with the same channel available, the more number of bands in which these channels locate, the less throughput can be achieved. The reason is that more bands cause more cost in performing CA. However, as the number of the small cells increases, the gap between the red line and the blue line decreases. Especially for the small cell network with 35 or more cells, the average throughput is about the same for $M=[3,3]$ and $M=[2,2,2]$. The reason is that as the density of small cells becomes larger, the mutual interference is more severe which plays a more important part than inter-band cost in selecting channels for CA. And when small cells are denser enough, e.g., there are 35 or more cells in the network, the mutual interference is so severe that the inter-band cost is negligible according to (7). In addition, the network performance for $M=[3,3]$ is significantly improved compared with $M=[2,2]$ and the gap between the blue line and the black line is almost uniform for different number of cells. It is rational that the more channels are available, the more throughput can be achieved correspondingly. The presented results also imply that searching more available spectrum resources is a good way to improve the network capacity. 
In Fig. 7, we compare the throughput performance under different $\delta$. From the simulation result it is noted that as $\delta$ increases the expected average throughput for each small cell decreases firstly and then increases. The reason is that when $\delta$ is less than 2.5, it is beneficial to aggregate a white channel according to the defined utility function (6), but larger $\delta$ takes more cost which make the throughput less according to equation (2). And when $\delta$ outnumbers 2.5, it is not beneficial to aggregate a white channel according to (6), it is more likely that small cells aggregate intra-band channels rather than inter-band channels. Consequently, the throughput increases. Besides, when $\delta=0$, such an CA model has also been applied for multiple channel selection in small cell networks.

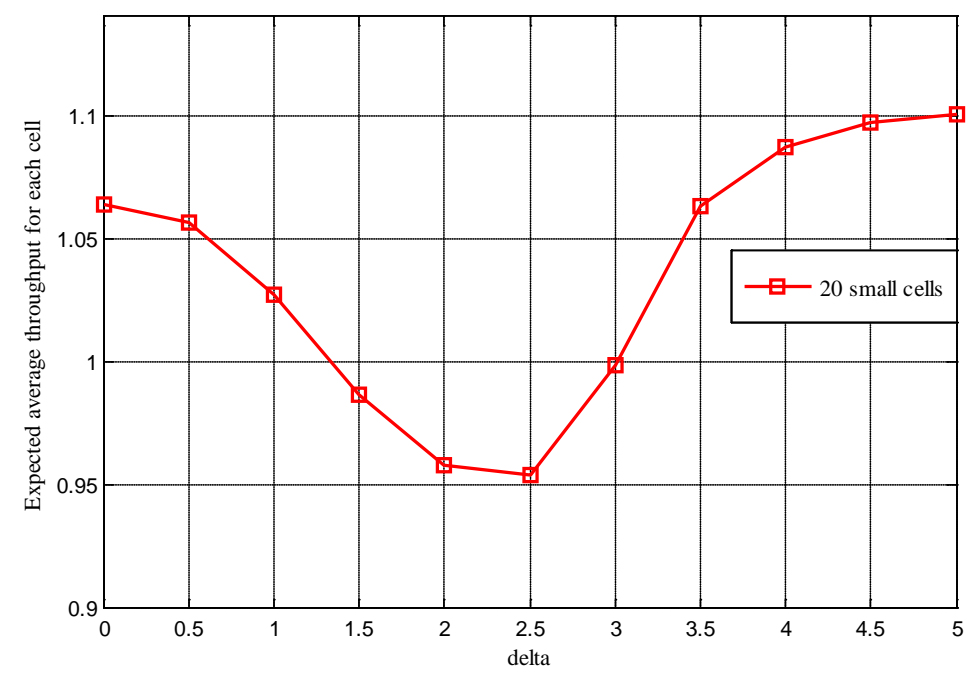

Fig. 7. The expected average network throughput for each small cell when varying the degree of flexibility in performing CA, i.e., $\delta$ (larger $\delta$ means that greater cost would be paid for performing

$$
\text { CA) }\left(B=3, K_{b}=2, M_{i}=3, b_{M}=3\right)
$$

\section{Conclusion}

To cope with the local interference and the distinct cost of intra-band and inter-band CA, we proposed a non-cooperation game which was proved to be an exact potential game. Furthermore, we proposed a spatial adaptive play algorithm with heterogeneous learning parameters (SAP-H) to converge towards NE of the game. In this algorithm, heterogeneous learning parameters were introduced to accelerate the convergence speed. It is shown that with the proposed game-theoretic approach, global optimization is achieved with local information. Simulation results validated the effectivity of the proposed game-theoretic CA approach. In addition, we studied other factors to affect the network throughput in performing CA and obtained some meaningful conclusions. In future, we plan to study QoS-aware CA in heterogeneous wireless communication system. 


\section{References}

[1] S. Chou, T. Chiu, Y. Yu and A. Pang, "Mobile small cell deployment for next generation cellular networks,” IEEE Global Communications Conference (GLOBECOM), pp. 4852-4857, December 8-12, 2014. Article (CrossRef Link).

[2] J. G. Andrews, S. Buzzi, Wan Choi and S. V. Hanly, "What will 5G be?” IEEE Journal on Selected Areas in Communications, vol. 32, no. 6, pp. 1065-1082, June, 2014. Article (CrossRef Link).

[3] Minho Jo, T. Maksymyuk, B. Strykhalyuk, Choong-Ho Cho, "Device-to-device-based heterogeneous radio access network architecture for mobile cloud computing," IEEE Wireless Communications, vol. 22 , no. 3, pp. 50-58, June, 2015. Article (CrossRef Link).

[4] Minho Jo, T. Maksymyuk, R.L. Batista, T.F. Maciel, "A survey of converging solutions for heterogeneous mobile networks,” IEEE Wireless Communications, vol. 21 , no. 6, pp. 54-62, December, 2014. Article (CrossRef Link).

[5] Z. Khan, H. Ahmadi, E. Hossain and M. Coupechoux, "Carrier aggregation/channel bonding in next generation cellular networks: Methods and challenges,” IEEE Network, vol. 28, no. 6, pp. 34-40, December, 2014. Article (CrossRef Link).

[6] H. Ahmadi, I. Macaluso and L. A. DaSilva, "Carrier aggregation as a repeated game: Learning algorithms for convergence to a Nash equilibrium,” in Proc. of IEEE Global Communications Conference (GLOBECOM), pp. 1233-1239, December 9-13, 2013. Article (CrossRef Link).

[7] M. Iwamura, K. Etemad, M. Fong and R. Nory, "Carrier aggregation framework in 3GPP LTE-Advanced,” IEEE Communications Magazine, vol. 48, no. 8, pp. 60-67, August, 2010. Article (CrossRef Link).

[8] Ngo, D. Trong, Le-Ngoc, Tho, "Architectures of small-cell networks and interference management," SpringerBriefs in Computer Science, Springer International Publishing, 2014. Article (CrossRef Link).

[9] A. Checko, H. L. Christiansen, Y. Yany, et al., "Cloud RAN for mobile networks: A technology overview," IEEE Communications Surveys and Tutorials, vol. 17, no. 1, pp. 405-426, September, 2014. Article (CrossRef Link).

[10] Y. Dai, A. Cuffaro, J. Gauvreau and R. Di Girolamo, "Dynamic spectrum management system aggregating non-contiguous spectrum over TVWS," in Proc. of 7th International ICST Conference on Cognitive Radio Oriented Wireless Networks and Communications (CROWNCOM), pp. 303-308, June 18-20, 2012. Article (CrossRef Link).

[11] A. M. Ghaleb, E. Yaacoub and A. A. Abdulkafi, "On the extension of traditional resource allocation algorithms in LTE-A to joint UL-DL scheduling with FDD carrier aggregation,” in Proc. of IEEE Symposium on Wireless Technology and Applications (ISWTA), pp. 70-74, Sept. 28-Oct. 1, 2014. Article (CrossRef Link).

[12] O. N. Gharehshiran, V. Krishnamurthy and G. Yin, "Distributed energy-Aware diffusion least mean squares: Game-theoretic learning,” IEEE Journal of Selected Topics in Signal Processing, vol. 7, no. 5, pp. 821-836, June, 2013. Article (CrossRef Link).

[13] Y. Xiao, G. Bi, D. Niyato and L. A. DaSilva, "A hierarchical game theoretic framework for cognitive radio networks,” IEEE Journal on Selected Areas in Communications, vol. 30, no. 10, pp. 2053-2069, November, 2012. Article (CrossRef Link).

[14] M. Lauridsen, H. Wang and P. Mogensen, "LTE UE energy saving by applying carrier aggregation in a HetNet scenario,” in Proc. of IEEE 77th Vehicular Technology Conference (VTC Spring), pp. 1-5, June 2-5, 2013. Article (CrossRef Link).

[15] F. Chung, J. Li, C. Chi and C. Lai, "Design of carrier-aggregated modulator for LTE-Advanced," in Proc. of IEEE MTT-S International Microwave Symposium (IMS), pp. 1-3, June 1-6, 2014. Article (CrossRef Link).

[16] M. Li, M. El-Hakiki, D. Kalim and Tae-Young Kim, “A fully matched LTE-A carrier aggregation quadplexer based on BAW and SAW technologies," in Proc. of IEEE International Ultrasonics Symposium (IUS), pp. 77-80, September 3-6, 2014. Article (CrossRef Link).

[17] S. C. Hwu and B. Razavi, “An RF receiver for intra-band carrier aggregation,” IEEE Journal of Solid-State Circuits, vol. 50 , no. 4, pp. 946-961, February, 2015. Article (CrossRef Link). 
[18] P. Ren, Y. Wang and Q. Du, "CAD-MAC: A channel-aggregation diversity based MAC protocol for spectrum and energy efficient cognitive Ad Hoc networks," IEEE Journal on Selected Areas in Communications, vol. 32, no. 2, pp. 237-250, May, 2013. Article (CrossRef Link).

[19] G. Yu, Q. Chen, R. Yin and H. Zhang, "Joint downlink and uplink resource allocation for energy-efficient carrier aggregation,” IEEE Transactions on Wireless Communications, vol. 14, no. 6, pp. 3207-3218, February, 2015. Article (CrossRef Link).

[20] A. M. Ghaleb, E. Yaacoub and A. A. Abdulkafi, "QoS-aware joint uplink-downlink scheduling in FDD LTE-Advanced with carrier aggregation,” in Proc. of 11th International Symposium on Wireless Communications Systems (ISWCS), pp. 111-115, August 26-29, 2014. Article (CrossRef Link).

[21] H. Wang, C. Rosa and K. Pedersen, “Analysis of carrier deployment strategies for LTE-A HetNets with multicell cooperation,” in Proc. of IEEE 80th Vehicular Technology Conference (VTC Fall), pp. 1-5, September 14-17, 2014. Article (CrossRef Link).

[22] H. Wang, C. Rosa and K. I. Pedersen, "Uplink inter-site carrier aggregation between macro and small cells in heterogeneous networks," in Proc. of IEEE 80th Vehicular Technology Conference (VTC Fall), pp. 1-5, September 14-17, 2014. Article (CrossRef Link).

[23] K. Mori, H. Nakamura, K. Naito and H. Kobayashi, "Inter-cell carrier aggregation for next generation mobile systems with non-uniform traffic," in Proc. of 2012 IEEE Region 10 Conference (TECON2012), pp. 1-6, November 19-22, 2012. Article (CrossRef Link).

[24] G. Yoshizaki, K. K. Mori, Naito and H. Kobayashi, "Enhanced inter-cell carrier aggregation with spread spectrum technique for next generation mobile systems," in Proc. of 21st International Conference on Telecommunications (ICT), pp. 379-383, May 4-7, 2014. Article (CrossRef Link).

[25] F. Palmieri, Ugo Fiore, S. Ricciardi, "Selfish routing and wavelength assignment strategies with advance reservation in inter-domain optical networks," Computer Communications, vol. 35, no. 3, pp. 366-379, February, 2012. Article (CrossRef Link).

[26] W. Wang, A. Kwasinski, Z. Han, "A routing game in cognitive radio networks against routing-toward-primary-user attacks," IEEE Wireless Communications and Networking Conference (WCNC), pp. 2510-2515, April 6-9, 2014. Article (CrossRef Link).

[27] Y. Xiao, T. Forde, I. Macaluso, L. DaSilva and L. Doyle, "Spatial spectrum sharing-based carrier aggregation for heterogeneous networks," in Proc. of IEEE Global Telecommunications Conference (GLOBECOM 2012), pp. 2615-2620, December 3-7, 2012. Article (CrossRef Link).

[28] Y. Xiao, C. Yuen, P. Di Francesco and L. DaSilva, "Dynamic spectrum scheduling for carrier aggregation: A game theoretic approach,” in Proc. of 2013 IEEE International Conference on Communications (ICC), pp. 2672-2676, June 9-13, 2013. Article (CrossRef Link).

[29] Q. Zhang, L. Guo, C. Sun and X. An, "Joint power control and component carrier assignment scheme in heterogeneous network with carrier aggregation,” IET Communications, vol. 8, no. 10, pp. 1831-1836, July, 2014. Article (CrossRef Link).

[30] P.T. Semov, A. Mihovska and R. Prasad, "Increasing throughput and fairness for users in heterogeneous semi coordinated deployments,” IEEE Wireless Communications and Networking Conference Workshops (WCNCW), pp. 40-45, April 6-9, 2014. Article (CrossRef Link).

[31] X. Chen and J. Huang, "Database-assisted distributed spectrum sharing,” IEEE Journal on Selected Areas in Communications, vol. 31, no. 11, pp. 2349-2361, May, 2013. Article (CrossRef Link).

[32] Y. Xu, J. Wang, Q. Wu and A. Anpalagan, “Opportunistic spectrum access in cognitive radio networks: global optimization using local interaction games,” IEEE Journal of Selected Topics in Signal Processing, vol. 6, no. 2, pp. 180-194, March, 2012. Article (CrossRef Link).

[33] D. Monderer and L. S. Shapley, "Potential games," Games and Economic Behavior, vol. 14, pp. 124-143, May, 1996. Article (CrossRef Link).

[34] J. Marden, G. Arslan, and J. Shamma, “Cooperative control and potential games," IEEE Transactions on Systems, Man, and Cybernetics, Part B: Cybernetics, vol. 39, no. 6, pp. 1393-1407, April, 2009. Article (CrossRef Link).

[35] H. P. Young, "Individual strategy and social structure," Princeton, NJ: Princeton Univ. Press, 1998. 
[36] R. Myerson, “Game theory: Analysis of conflict,” Cambridge, MA: Harvard Univ. Press, 1991.

[37] Y. Xu, Q. Wu, J. Wang, L. Shen and A. Anpalagan, “Opportunistic spectrum access using partially overlapping channels: Graphical game and uncoupled learning," IEEE Transactions on Communications, vol. 61, no. 9, pp. 3906-3918, August, 2013. Article (CrossRef Link).
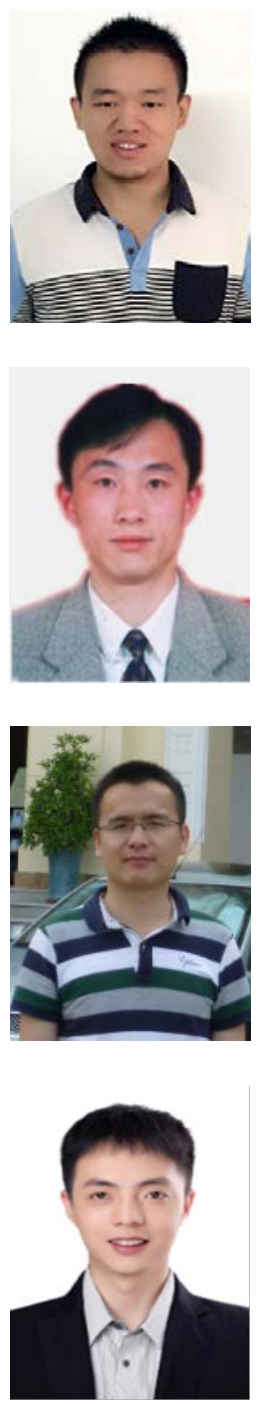

Yuanhui Zhang received his B.S. degree in applied physics from college of science, National University of Defense Technology, Changsha, China, in 2009. He is currently pursuing the M.S. degree in information and communications engineering in Institute of Communications Engineering, PLA University of Science and Technology. His research interests focus on carrier aggregation, LTE-U, game theory, and dynamic spectrum access.

Chunrong Kan received his B.S. degree in communications engineering, M.S. degree and Ph.D. degree in communications and information system from Institute of Communications Engineering, Nanjing, China, in 1993 , 2001, and 2006 respectively. He is currently an associate professor at the PLA University of Science and Technology, China. His current research interests are algorithms for soft-defined radio and wireless communication systems.

Kun Xu was born in Fengcheng, Jiangxi Province, China, on April 5, 1986. He received the B.S. degree in communications engineering and Ph.D. degree in communications and information system from College of Communications Engineering, PLA university of Science and Technology(PLAUST), Nanjing, China, in 2007 and in 2013, respectively. His research interests include cooperative communications, cognitive radios, MIMO and HF communications. He is now working as a teacher in PLA Academy of National Defense Information, Wuhan, China.

Yuhua Xu received his B.S. degree in Communications Engineering, and Ph.D. degree in Communications and Information Systems from College of Communications Engineering, PLA University of Science and Technology, in 2006 and 2014 respectively. He has been with College of Communications Engineering, PLA University of Science and Technology since 2012, and currently as an Assistant Professor. His research interests focus on opportunistic spectrum access, learning theory, game theory, and distributed optimization techniques for wireless communications. He has published several papers in international conferences and reputed journals in his research area. He is an Editor for the KSII Transactions on Internet and Information Systems. In 2011 and 2012, he was awarded Certificate of Appreciation as Exemplary Reviewer for the IEEE Communications Letters. 